Wijnhoven, T.; Deconinck, G.; Neumann, T.; Erlich, I., "Control aspects of the dynamic negative sequence current injection of type 4 wind turbines," in IEEE PES General Meeting, Washington DC, 27-31 July 2014, 5 p.

doi: 10.1109/PESGM.2014.6938931

URL: $\underline{\text { http://ieeexplore.ieee.org/stamp/stamp.jsp?tp=\&arnumber=6938931\&isnu }}$ $\underline{\mathrm{mber}}=6938773$

(c) 2014 IEEE. Personal use of this material is permitted. Permission from IEEE must be obtained for all other users, including reprinting/ republishing this material for advertising or promotional purposes, creating new collective works for resale or redistribution to servers or lists, or reuse of any copyrighted components of this work in other works.

(below follows the accepted version) 


\section{Control Aspects of the Dynamic Negative Sequence Current Injection of Type 4 Wind Turbines}

\author{
Thomas Wijnhoven \\ Geert Deconinck \\ KU Leuven \\ Leuven, Belgium
}

\author{
Tobias Neumann \\ István Erlich \\ University of Duisburg-Essen \\ Duisburg, Germany
}

\begin{abstract}
State-of-the-art distributed generation with a fully rated converter only injects positive sequence current into unbalanced faults. Recently, both publications and draft standards have suggested that the injection of negative sequence reactive current into unbalanced faults can have benefits for the power system. This paper discusses a selection of control aspects that are important for negative sequence current injection: negative sequence angle detection, converter current limitation, and voltage recovery after fault clearing. The impact of these control aspects, and negative sequence current injection in general, on the power system is also considered in the discussion.
\end{abstract}

Index Terms-Control, Converter, Negative Sequence, Wind Power

\section{INTRODUCTION}

As more and more distributed generation units (DG) are integrated in the power system, their fault behavior starts to play a bigger role in the system. Studies on this behavior mainly focus on three phase faults and grid codes usually only specify requirements for positive sequence current injections during faults. Conventional synchronous generators and stateof-the-art type 3 wind turbines (WT) with a doubly fed induction generator (DFIG) have a natural negative sequence current contribution during unbalanced faults through the direct connection of their stator with the power system [1]. However, type 4 WT with a fully rated converter don't have a natural negative sequence current contribution as their positive and negative sequence behavior is determined by the converter controls. Consequently, state-of-the-art type 4 WT only inject positive sequence current into faults, regardless whether it is a balanced or an unbalanced fault. Recently, there has been given more attention to the possibility of injecting negative sequence current into unbalanced faults [2], [3]. In Germany, a draft standard already suggests a reactive negative sequence current contribution during negative sequence grid voltages [4]. A proposal is illustrated in Figure 1 , where $k_{2}$ represents the gain of the dynamic current control loop for the negative sequence. The aim of the injected negative sequence inductive reactive current is to reduce the negative sequence voltage. Consequently, the DG will also deliver an unbalanced fault current contribution that is more similar to conventional synchronous generators.

This paper aims to clarify a number of control aspects related to negative sequence current injection. It is organized as follows: Section II gives a mathematical description of the concepts and representations of symmetrical components that are used in this paper. Next, section III gives a short overview of the controls for type $4 \mathrm{WT}$, although the principles are the same for any DG with a fully rated converter (e.g. PV) or any
Voltage Source Converter (VSC) based connection to the AC power system (e.g. HVDC transmission system). Section IV discusses several important control aspects of the negative sequence current injection. In addition, the principle of its voltage stabilizing effect is emphasized in a schematic example.

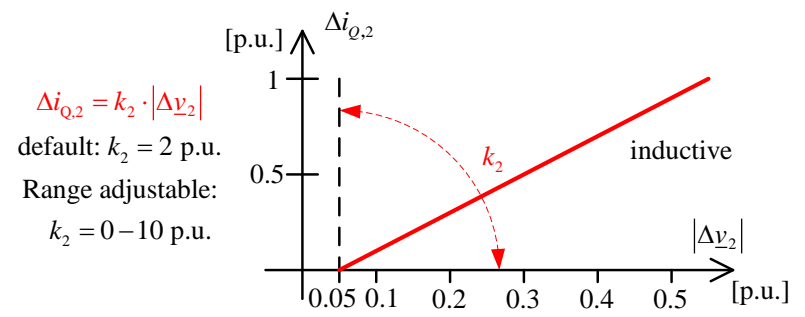

Figure 1: Injection of negative sequence reactive current in relationship with the negative sequence grid voltage

\section{MATHEMATICAL DESCRIPTION OF SYMMETRICAL COMPONENTS}

This section explains the concepts and representations of symmetrical components that are used in this paper. Symmetrical components are described in great detail in literature since the original paper of Fortescue [5]. A good overview of the symmetrical components in the time domain, including the relationship between symmetrical components, the Park transformation (to the synchronously rotating or d,q frame) and the Clarke transformation (to the stationary or $\alpha, \beta$ frame) is given in [6]. As this paper deals with three phase sources, without neutral connection, the sources cannot inject zero sequence currents. Therefore the description is limited to positive and negative sequence.

Figure 2 shows positive and negative sequence components and their composition to the total phase values (here of the voltage system) calculated based on the transformation matrix $\left(\underline{a}=\mathrm{e}^{\mathrm{j} \cdot\left(120^{\circ}\right)}\right)$

$$
\left[\begin{array}{l}
\underline{V}_{1} \\
\underline{V}_{2} \\
\underline{V}_{0}
\end{array}\right]=\frac{1}{3}\left[\begin{array}{ccc}
1 & \underline{\mathrm{a}} & \underline{\mathrm{a}}^{2} \\
1 & \underline{\mathrm{a}}^{2} & \underline{\mathrm{a}} \\
1 & 1 & 1
\end{array}\right]\left[\begin{array}{l}
\underline{V}_{\mathrm{a}} \\
\underline{V}_{\mathrm{b}} \\
\underline{V}_{\mathrm{c}}
\end{array}\right]
$$

For the time-varying abc voltages this results in:

$$
\begin{aligned}
& v_{\mathrm{a}}(t)=\sqrt{2}\left(V_{1} \cos \left(\omega t+\theta_{1}\right)+V_{2} \cos \left(\omega t+\theta_{2}\right)\right) \\
& v_{\mathrm{b}}(t)=\sqrt{2}\left(V_{1} \cos \left(\omega t-\frac{2 \pi}{3}+\theta_{1}\right)+V_{2} \cos \left(\omega t+\frac{2 \pi}{3}+\theta_{2}\right)\right) \\
& v_{\mathrm{c}}(t)=\sqrt{2}\left(V_{1} \cos \left(\omega t+\frac{2 \pi}{3}+\theta_{1}\right)+V_{2} \cos \left(\omega t-\frac{2 \pi}{3}+\theta_{2}\right)\right)
\end{aligned}
$$

with $V_{1 / 2}$ the amplitude of the positive/negative sequence 
voltage phasor. For the currents an analogous expression is valid, with other phase angles $\left(\theta_{1}+\theta_{1 \mathrm{i}} / \theta_{2}+\theta_{2 \mathrm{i}}\right.$ instead of $\left.\theta_{1} / \theta_{2}\right)$ and magnitudes $\left(I_{1} / I_{2}\right.$ instead of $\left.V_{1} / V_{2}\right)$.

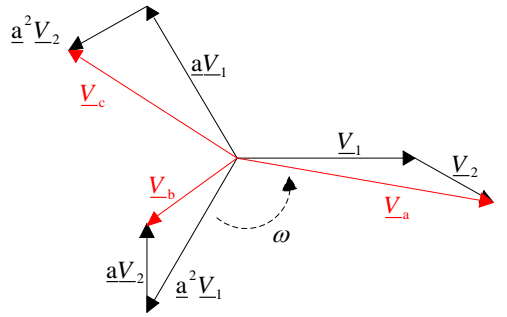

Figure 2: Positive and negative sequence components and their composition to the total phase values (without zero sequence)

In the following section the per-unit (p.u.) system represented in small letters with respect to the corresponding base quantity is used. The time-varying abc values can be transformed to a complex space vector in the stationary or $\alpha, \beta$ frame $(\angle 0)$ by using the Clarke transformation [7].

$$
\underline{v}^{\angle 0}=v_{\alpha}+\mathrm{j} v_{\beta}=2 / 3\left(v_{\mathrm{a}}+\underline{a} \cdot v_{\mathrm{b}}+\underline{a}^{2} \cdot v_{\mathrm{c}}\right)
$$

Under unbalanced conditions the total space vector consists of a positive and a negative sequence component.

$$
\begin{aligned}
\underline{v}^{\angle 0} & =1 / 3\left(\underline{v}_{\mathrm{a}}+\underline{v}_{\mathrm{a}}^{*}+\underline{a} \cdot\left(\underline{v}_{\mathrm{b}}+\underline{v}_{\mathrm{b}}^{*}\right)+\underline{a}^{2} \cdot\left(\underline{v}_{\mathrm{c}}+\underline{v}_{\mathrm{c}}^{*}\right)\right) \\
& =1 / 3\left(\underline{v}_{\mathrm{a}}+\underline{a} \cdot \underline{v}_{\mathrm{b}}+\underline{a}^{2} \cdot \underline{v}_{\mathrm{c}}\right)+\frac{1}{3}\left(\underline{v}_{\mathrm{a}}^{*}+\underline{a} \cdot \underline{v}_{\mathrm{b}}^{*}+\underline{a}^{2} \cdot \underline{v}_{\mathrm{c}}^{*}\right) \\
& =\underline{v}_{1}^{\angle 0}+\underline{v}_{2}^{*} \angle 0
\end{aligned}
$$

The positive sequence space vector rotates counterclockwise while the conjugate complex negative sequence space vector rotates in the opposite direction, as is illustrated in Figure 3 and Figure 4. In these figures the space vector of positive and negative sequence voltage in the $\alpha, \beta$ reference frame are aligned to the corresponding phase angle represented in the synchronously rotating reference frame.

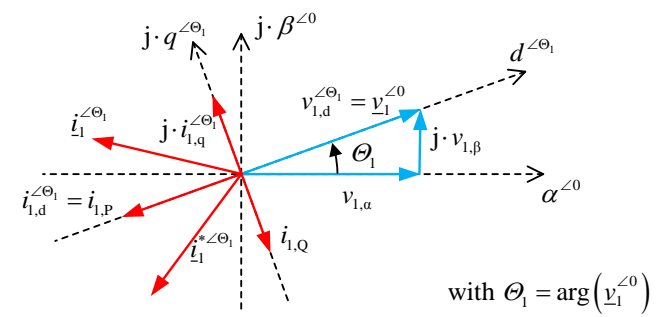

Figure 3: Positive sequence in $\alpha, \beta$ and $\mathrm{d}, \mathrm{q}$ frame

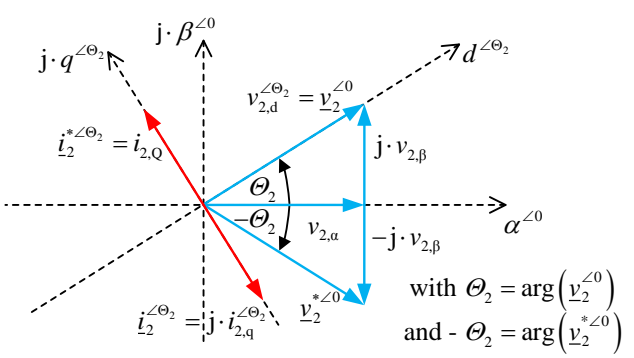

Figure 4: Negative sequence in $\alpha, \beta$ and $d, q$ frame

In an unbalanced system the p.u. instantaneous power in a three-phase system can be calculated from the space vectors of voltage and currents in an arbitrary reference system $(\angle)$ with

$$
\begin{aligned}
& \underline{s}=\underline{v}^{<} \cdot \underline{i}^{*}< \\
& =\left(\underline{v}_{1}^{<}+\underline{v}_{2}^{*<}\right) \cdot\left(\underline{i}_{1}^{*<}+\underline{i}_{2}^{<}\right)
\end{aligned}
$$

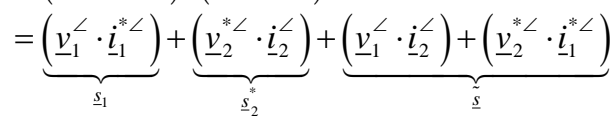

The positive and negative sequence apparent, active and reactive power can be written as

$$
\underline{s}_{1}=p_{1}+\mathrm{j} \cdot q_{1}=\underline{v}_{1}^{<} \cdot \underline{i}_{1}^{*<} \quad \& \quad \underline{s}_{2}=p_{2}+\mathrm{j} \cdot q_{2}=\underline{v}_{2}^{<} \cdot \underline{i}_{2}^{*<}
$$

with the corresponding active and reactive currents

$$
i_{1, \mathrm{P}}=p_{1} /\left|\underline{v}_{1}\right|, i_{1, \mathrm{Q}}=q_{1} /\left|\underline{v}_{1}\right| \quad \& \quad i_{2, \mathrm{P}}=p_{2} /\left|\underline{v}_{2}\right|, i_{2, \mathrm{Q}}=q_{2} /\left|\underline{v}_{2}\right|
$$

During unbalanced conditions both $\underline{\mathrm{s}}_{1}$ and $\underline{\mathrm{s}}_{2}$ are constant under steady state but the cross coupling of positive and negative sequence components of voltage and currents lead to an oscillating power component $\underline{\underline{s}}$. The frequency of this oscillating power is twice of the nominal frequency and it leads to the conclusion that oscillating power injections are in general unavoidable when the system voltages are unbalanced.

\section{CONTROL OVERVIEW OF TYPE 4 WIND TURBINES}

The type 4 WT concept is using either a synchronous or asynchronous generator, which is connected to the power system via a fully rated converter system. Therefore the generator is completely decoupled from the power system and only the line side converter (LSC) is essentially responsible for the dynamic response of the WT during grid faults.

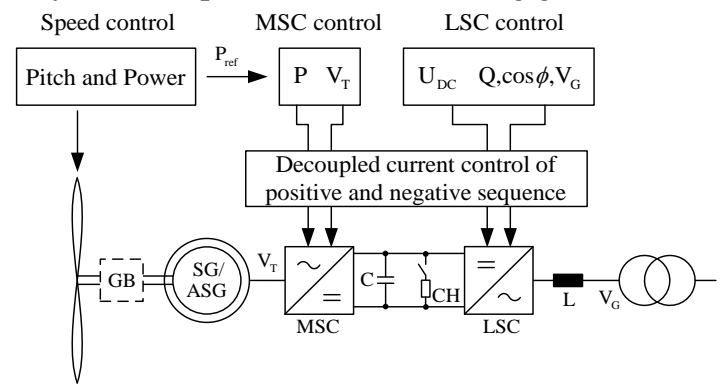

Figure 5: Generator with Fully Rated Converter

In this concept, depicted in Figure 5, the machine side converter (MSC) functions as a rectifier and does not necessarily require a decoupled control of positive and negative sequence. By contrast, the LSC is usually equipped with a decoupled control of active and reactive power (active power is controlled via the DC voltage) in the positive sequence and a parallel negative sequence control loop. The LSC allows a full controllability in the positive and in the negative sequence within its current limitation. The entire block diagram of the positive and negative sequence current control loops are presented in [2].

\section{CONTROL ASPECTS OF DG WITH FULLY RATED CONVERTER}

This section discusses three main control aspects:

- Negative sequence angle detection and timing of negative sequence current injection

- Influence of negative sequence current injection on the DG current limitation and comparison with balanced faults

- Negative sequence current injection influence on voltage recovery after the fault 


\section{A. Negative sequence angle detection and timing of the negative sequence current injection}

During unbalanced and also during balanced conditions in the power system mathematical algorithms in the controller decompose the instantaneous values of voltage and currents in positive and negative sequence and control them separately and decoupled from each other (see section III). A full treatment of the algorithms for the separation of positive and negative sequence voltages is outside the scope of this paper, but several methods are described in literature [8]. After this separation, the phase angle of the negative sequence voltage can be calculated from its space vector (see Figure 4). In balanced conditions without negative sequence in the system, the magnitude of the negative sequence voltage space vector is very small and disturbed by noise of the measurements. Input filters are reducing the noise for an accurate utilization of the signals in the control loops but cannot avoid noise totally. With a small magnitude of the negative sequence voltage space vector the accurate phase angle calculation is problematic and sometimes even impossible.

As long as the control target of the negative sequence controller is eliminating the negative sequence current to zero,
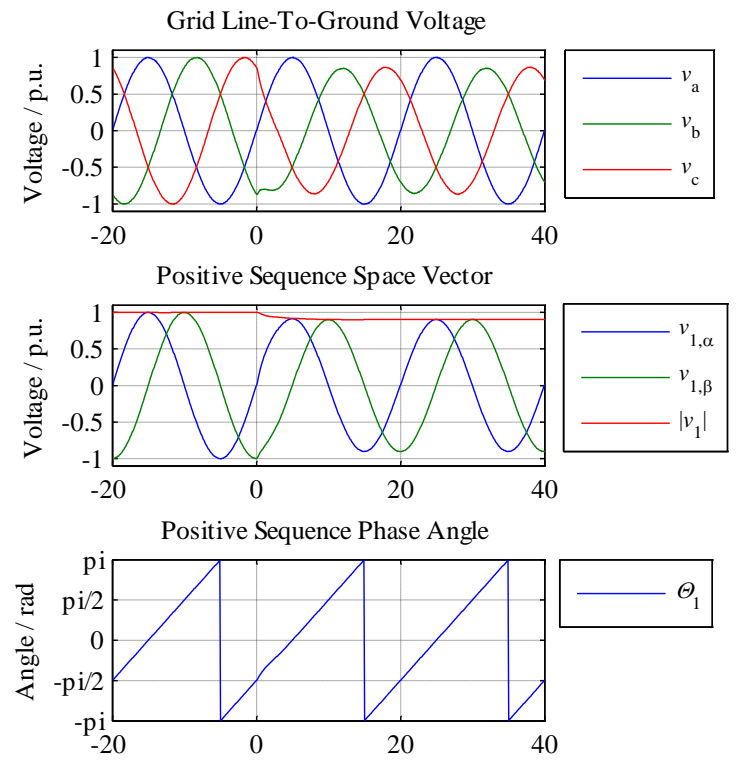

Conjugate Complex Negative Sequence Space Vector
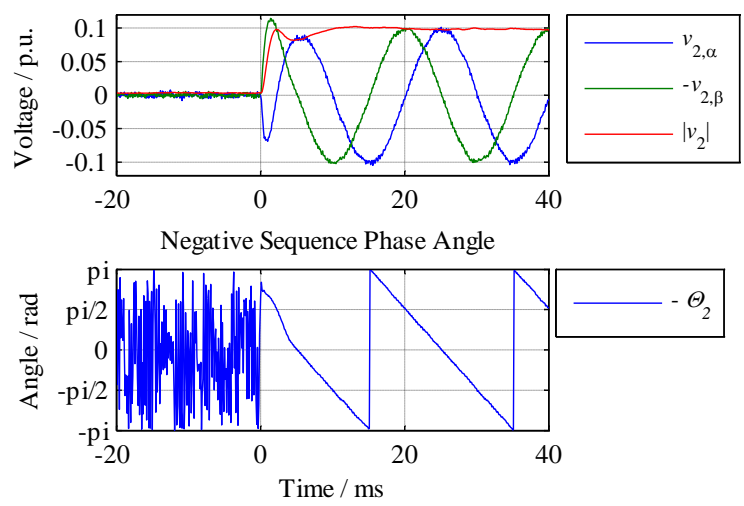

Figure 6: 10\% positive sequence voltage reduction and $10 \%$ negative sequence voltage increase at $0 \mathrm{~ms}$ the negative sequence control loop doesn't necessarily has to be aligned to the negative sequence phase angle. Because the positive sequence angular speed is the same as the native sequence angular speed, the positive sequence angle can be used as the reference angle for the transformation from the stationary to the rotating reference frame (dq- or Parktransformation) in the negative sequence control loop during balanced conditions.

By contrast for the dynamic injection of a certain negative sequence current during unbalanced fault conditions, detecting the phase angle of the negative sequence voltage must be performed fast and accurately in order to give the negative sequence current the correct alignment. This alignment is required to inject a negative sequence inductive reactive current and thus to achieve the goals of reducing the negative sequence voltage and providing a fault current contribution that is more similar to conventional generators. A wrong alignment could even worsen the situation.

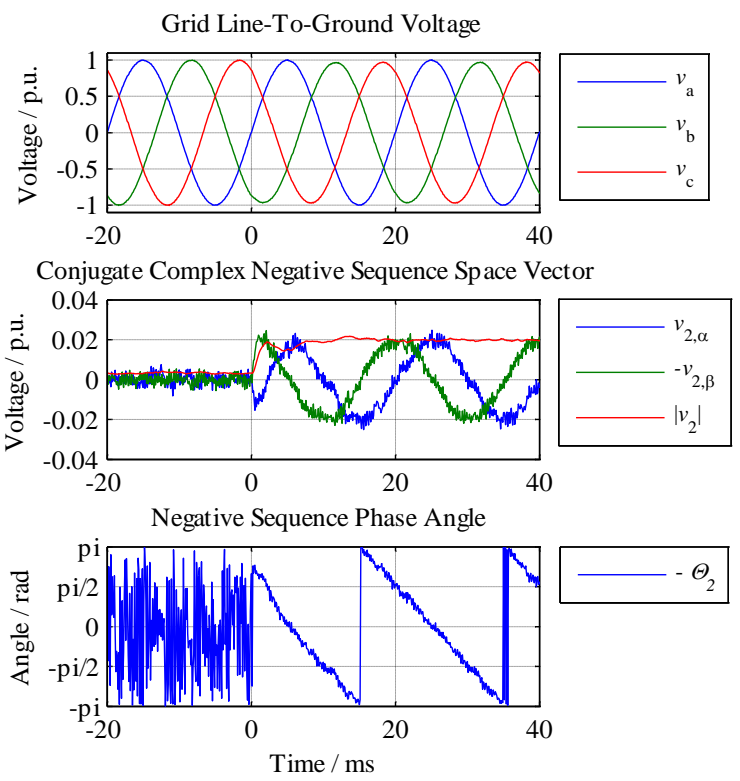

Figure 7: 2\% negative sequence voltage increase at $0 \mathrm{~ms}$

As a consequence a certain magnitude of the negative sequence voltage must exist for a correct negative sequence current injection which leads to the implementation of a deadband for the dynamic negative sequence control. Figure 6 and Figure 7 show simulation results for far away line-to-line grid faults which lead to a decrease in the positive sequence voltage and an increase of the negative sequence voltage. In the figures the measured, internal voltage signals of a simulated low voltage converter system are visualized (for Figure 7 the positive sequence signals are omitted as they are similar to the ones in Figure 6). In the first example the conjugate complex negative sequence space vector has a magnitude of 0.1 p.u. which leads to an accurate calculation of the negative sequence phase angle. In the second example the magnitude of the negative sequence space vector is limited to 0.02 p.u. As a result the negative sequence angle calculation has undesirable noise on the signal which leads to an inaccurate control of the negative sequence current injection.

A deadband of the negative sequence voltage of at least 0.05 p.u. seems to be a good compromise (cf. Figure 1). 
Additionally, Figure 6 illustrates another aspect: an accurate estimation of the negative sequence angle requires a quarter to half of a period (5-10 ms at $50 \mathrm{~Hz}$ ) because the information in the sine wave is only fully known after that period of time.

\section{$B$. Influence of negative sequence current injection on the} DG current limitation

For a type 4 converter the total current contribution is limited by the converter rating. Injecting negative sequence current in addition to positive sequence current will limit the amount of positive sequence current that can be injected. However a straightforward limitation that is used in [2]:

$$
\left|\underline{i}_{1}\right|+\left|\underline{i}_{2}\right| \leq i_{\max }
$$

with $\underline{i}_{1}$ and $\underline{i}_{2}$ the positive/negative sequence phasors in the rotating d,q frames, is in general too severe. The limitation of the converter is valid in the abc frame, so a more advanced limitation should be applied in the abc frame. This limitation is based on [9], but the notation of section II is used.

$$
\begin{aligned}
& i_{\mathrm{a}-\max }=\sqrt{i_{1}^{2}+i_{2}^{2}+2 i_{1} i_{2} \cos \alpha} \\
& i_{\mathrm{b}-\max }=\sqrt{i_{1}^{2}+i_{2}^{2}+2 i_{1} i_{2} \cos \left(\alpha+\frac{4 \pi}{3}\right)} \\
& i_{\text {c-max }}=\sqrt{i_{1}^{2}+i_{2}^{2}+2 i_{1} i_{2} \cos \left(\alpha-\frac{4 \pi}{3}\right)}
\end{aligned}
$$

with $\alpha=\left(\theta_{2}+\theta_{2 \mathrm{i}}\right)-\left(\theta_{1}+\theta_{1 \mathrm{i}}\right)$

It is seen that the maximum current depends on the value of $\alpha$. For $\alpha=0,2 \pi / 3,4 \pi / 3$ the maximum current is equal to the one obtained by the straightforward limitation. For $\alpha=\pi / 3, \pi, 5 \pi / 3$ however, the advanced current limitation allows for a current that is $2 / \sqrt{ } 3$ or $15.4 \%$ bigger than the current that is allowed with the straightforward limitation.

As an illustration of the benefits of negative sequence injection and the possible gain of the advanced current limitation, a simple example is described below. In this example only (positive and negative sequence) reactive current is injected into the fault, although in general active current could also be injected. This choice between active and reactive current and the priority between both depends on the grid requirements. A discussion on these alternatives is outside the scope of this paper.

With the same gain of the dynamic current control loop for positive and negative sequence $\left(k_{1}=k_{2}\right.$, cf. Figure 1$)$, even the straightforward limitation will always limit the voltage dip in the positive sequence during a line-to-line fault to half the voltage dip during a three phase fault. To support this statement, a very general example is chosen. Consider the positive sequence scheme in Figure 8 where all values are expressed in p.u. The DG current limit is considered to be 1 p.u., although it can be designed for a slightly larger current. The gains of the control loops ( $k_{1}$ and $\left.k_{2}\right)$ are set to 2 p.u., so the DG will inject a reactive current of 1 p.u. into the fault if the voltage at its terminals equals 0.5 p.u. The impedance between the DG unit and the fault is a purely reactive impedance of 0.5 p.u. The resulting voltage dip at the location of the DG is $50 \%$.

In Figure 9, a line-to-line fault is applied at the same location. Both positive and negative sequence schemes are shown. For a gain $k_{1}=k_{2}=2$ p.u., the currents will be as shown on the figure, resulting in a positive sequence voltage of 0.75 p.u. at the terminals of the DG unit and thus a voltage dip in the positive sequence of $25 \% . \quad\left(v_{\mathrm{a}}=1 \mathrm{e}^{\mathrm{j} \cdot\left(0^{\circ}\right)}\right.$ p.u., $v_{\mathrm{b}}=0.66$ $\mathrm{e}^{\mathrm{j} .\left(-139^{\circ}\right)}$ p.u., $v_{\mathrm{c}}=0.66 \mathrm{e}^{\mathrm{j} .\left(139^{\circ}\right)}$ p.u.) This reduced voltage dip can be explained by the fact that for a perfect three phase fault, the positive sequence voltage can drop to 0 p.u. For a perfect lineto-line fault, the positive sequence voltage drops to 0.5 p.u. and the negative sequence voltage rises to 0.5 p.u. This leaves some margin for negative sequence current injection.

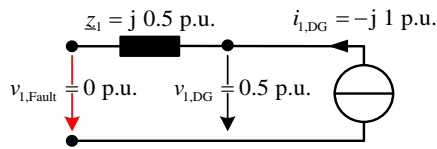

Figure 8: Three phase fault, maximum current contribution of DG

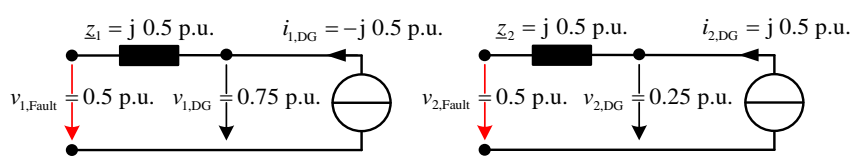

Figure 9: Line-to-line fault, maximum current contribution of DG with the straightforward limitation

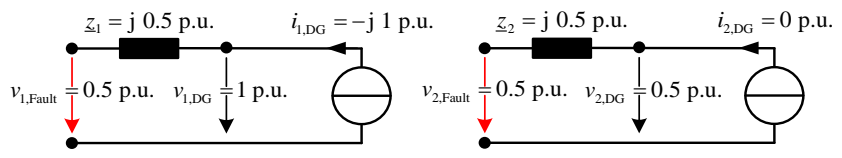

Figure 10: Line-to-line fault, maximum current contribution of DG with only positive sequence current injection (if gain $k_{1}>>2$ )

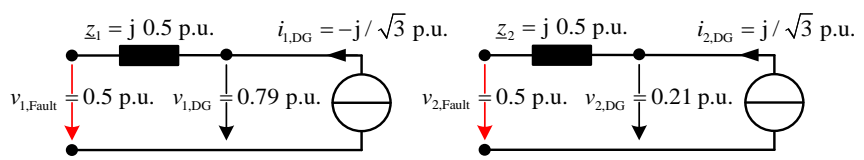

Figure 11: Line-to-line fault, maximum current contribution of DG with the advanced limitation (if gain $k_{1}=k_{2}>>2$ )

To illustrate the benefits of this negative sequence current injection, Figure 10 shows the maximum current contribution when only positive sequence current is injected. It is clear that an overvoltage results from these injections: $\left(v_{\mathrm{a}}=1.5 \mathrm{e}^{\mathrm{j} \cdot\left(0^{\circ}\right)}\right.$ p.u., $v_{\mathrm{b}}=0.87 \mathrm{e}^{\mathrm{j} \cdot\left(-150^{\circ}\right)}$ p.u., $v_{\mathrm{c}}=0.87 \mathrm{e}^{\mathrm{j} \cdot\left(150^{\circ}\right)}$ p.u. $)$ In addition, the phase angles have a larger deviation when compared with the negative sequence current injections.

The maximum current contribution for the advanced current limitation is shown in Figure 11. This illustrates that the advanced current limitation can obtain higher current injections and a more balanced voltage system without violating the DG current limits: $\left|i_{\mathrm{a}}\right|=0$ p.u., $\left|i_{\mathrm{b}}\right|=1$ p.u., $\left|i_{c}\right|=1$ p.u. $\quad\left(v_{\mathrm{a}}=1 \mathrm{e}^{\mathrm{j} \cdot\left(0^{\circ}\right)}\right.$ p.u., $\quad v_{\mathrm{b}}=0.71 \mathrm{e}^{\mathrm{j} .\left(-\left(135^{\circ}\right)\right.}$ p.u., $v_{\mathrm{C}}=0.71 \mathrm{e}^{\mathrm{j} .\left(135^{\circ}\right)}$ p.u.).

\section{Negative sequence current injection influence on voltage} recovery after the fault

Another aspect that needs to be considered for the dynamic negative sequence control is the effect of negative sequence current injection during voltage recovery. The transient period between fault and stable post fault conditions seems to be critical with respect to the voltage quality because both the negative and the positive sequence reactive current injections for the voltage support in the corresponding sequence always have a small delay. As a consequence, the injected currents will influence the voltages that are measured by the DG and will complicate the fast estimation of the new positive and negative sequence voltages which can lead to inaccurate 
control actions. In addition, the mathematical algorithms for decomposing the instantaneous values into symmetrical components have a delay in the range of a quarter to half of a period (5-10 ms at $50 \mathrm{~Hz}$ ) because the information in the sine wave is only fully known after that period of time, as was also mentioned in section IV.A.
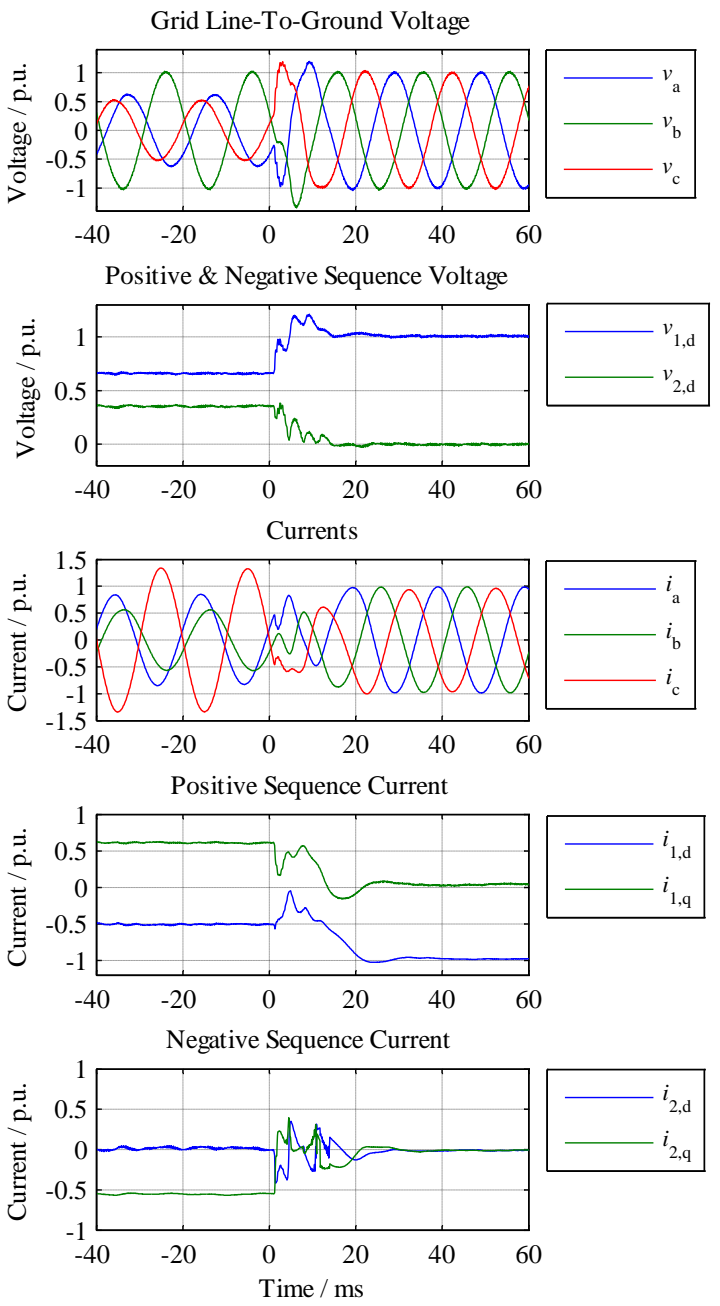

Figure 12: Voltages and currents during the voltage recovery of a type 4 wind turbine

Figure 12 shows the response of a type 4 WT during voltage recovery of a line-to-line fault. During this fault there is a reactive current injection in the positive sequence, represented by $i_{1, q}$ (capacitive), as well as in the negative sequence, represented by $i_{2, q}$ (inductive), and the active current is reduced to keep the inverter current within its limits (here 1.4 p.u.) using the straightforward limitation mentioned in section IV.B. After fault clearing both reactive currents are reduced to zero while the active currents in the positive sequence is ramped up to its pre-fault value of 1 p.u. The instantaneous values of voltage and currents show that the transient period of recovery takes roughly $10 \mathrm{~ms}$. The shape of the voltage during this period is very fault-specific and depends on the grid topology and the connected generation units.
From a controller point of view, there is no reason to have a deadband in the positive sequence current controller as the positive sequence angle can be estimated accurately near the normal operating points of the DG. However, for the negative sequence current controller it is recommended to reduce the reactive current injection immediately after the voltage of the negative sequence returns in its deadband. This reduces any adverse effects of the negative sequence current injection during voltage recovery and avoids problems with the alignment of the negative sequence current as was discussed in section IV.A.

\section{SUMMARY AND CONCLUSIONS}

From the discussion in this paper, it is clear that there are several control aspects that influence the negative sequence current injection of type 4 wind turbines during faults. The negative sequence angle estimations always have a small delay, which means that the negative sequence current injection will have a small delay of about half a period $(10 \mathrm{~ms}$ at $50 \mathrm{~Hz}$ ) because an accurate angle is required to inject the correct negative sequence current. When the fault is cleared, this delay also has an influence on the voltage recovery after the fault. The injection of negative sequence current also results in a lower positive sequence current injection capability. A schematic example illustrated that even with this limitation the injection of negative sequence currents into a line-to-line fault improves the voltages and results in a fault current contribution that resembles more the fault current contribution of conventional generation.

Future work will focus on the determination of optimal negative sequence current contributions based on grid requirements.

\section{REFERENCES}

S. Engelhardt, J. Kretschmann, J. Fortmann, F. Shewarega, I. Erlich, and C. Feltes, "Negative sequence control of DFG based wind turbines," in IEEE PES General Meeting, 2011.

I. Erlich, T. Neumann, F. Shewarega, P. Schegner, and J. Meyer, "Wind Turbine Negative Sequence Current Control and its Effect on Power System Protection," in IEEE PES General Meeting, 2013.

T. Wijnhoven and G. Deconinck, "Flexible fault current contribution with inverter interfaced distributed generation," in IEEE PES General Meeting, 2013.

VDE, "E VDE-AR-N 4120:2012-11 Technische Bedingungen für den Anschluss und Betrieb von Kundenanlagen an das Hochspannungsnetz." .

C. L. Fortescue, "Method of Symmetrical Co-Ordinates Applied to the Solution of Polyphase Networks," Trans. Am. Inst. Electr. Eng., vol. 37, pp. 1027-1140, Jul. 1918.

G. C. Paap, "Symmetrical components in the time domain and their application to power network calculations," IEEE Trans. Power Syst., vol. 15, pp. 522-528, May 2000.

C. Feltes, "Advanced Fault Ride-Through Control of DFIG based Wind Turbines including Grid Connection via VSC-HVDC,” Ph.D. dissertation, Universität Duisburg-Essen, 2011.

R. Teodorescu, M. Liserre, and P. Rodriguez, Grid Converters for Photovoltaic and Wind Power Systems. John Wiley and Sons, 2011, p. 416.

C.-T. Lee, C.-W. Hsu, and P.-T. Cheng, “A Low-Voltage RideThrough Technique for Grid-Connected Converters of Distributed Energy Resources,” IEEE Trans. Ind. Appl., vol. 47, pp. 18211832, Jul./Aug. 2011. 